\title{
Direct simulations of fluid dynamic sounds by the finite difference lattice Boltzmann method
}

\author{
M. Tsutahara, A. Tamura, S. Tajiri \& W. Long \\ Graduate School of Science and Technology, Kobe University, \\ Rokko, Nada, Kobe, Japan
}

\begin{abstract}
In this paper we present some applications of the finite difference lattice Boltzmann method (FDLBM) to direct simulations of fluid dynamic sound. The Arbitrary Lagrangian Eulerian formulation is introduced to FDLBM and the sounds emitted from moving bodies are successfully simulated. The two-particle model is used to simulate two-phase flows, and introducing a fluid elasticity the sound propagation inside the liquid is simulated. The sounds generated on the interface between the liquid and gas are also successfully simulated.
\end{abstract}

Keywords: fluid dynamic sound, lattice Boltzmann method, Arbitrary Lagrangian Eulerian formulation, two-phase flow, under water sound.

\section{Introduction}

The lattice Boltzmann method [1-6] is now a very powerful tool of computational fluid dynamics (CFD). This method is different from ordinary Navier-Stokes equations based CFD methods, and is based on the particle motions. However, mostly successful model so far is for incompressible fluids, but several models for thermal compressible models have been proposed including our model [7-13]. On the other hand, this method has great advantage to simulate multi-phase flows, because the interface is automatically determined in this method without special treatment [14-17].

We use a compressible fluid model of LBM and perform direct simulations of aerodynamic sound emitted from moving bodies using the arbitrary Lagrangian Eulerian formulation, especially the sound sources are detected. We also propose a new model for liquids considering the elasticity of liquid, and the sound speed propagating inside the liquid is correctly realized. A simulation of a water drop colliding the water surface and sound emission is performed. 


\section{Discrete BGK equation}

In this paper, we apply the discrete BGK equation as a governing equation of the FDLBM. The discrete BGK equation represents evolution of a velocity distribution function of particles $f_{i}(\mathbf{x}, t)$ as

$$
\frac{\partial f_{i}(\mathbf{x}, t)}{\partial t}+c_{i \alpha} \frac{\partial f_{i}(\mathbf{x}, t)}{\partial x_{\alpha}}=-\frac{1}{\tau}\left\{f_{i}(\mathbf{x}, t)-f_{i}^{(0)}(\mathbf{x}, t)\right\}
$$

where $t$ and $\boldsymbol{x}$ indicate the time and the space, respectively. Subscript $i$ and $\alpha$ denote directions of particles' motion and the space directions in Cartesian coordinates, and $c_{i \alpha}$ represents a particle velocity. $\tau$ is called a relaxation time and $f_{i}^{(0)}(\mathbf{x}, t)$ is the equilibrium distribution function, that is determined so as to recover the corresponding fluid dynamic equations. The details are shown later. The right hand side is a collision term, and represents that a particle distribution approaches an equilibrium state by the collisions among particles.

We introduce a particle model into equation (1) in order to determine the particle velocity and the equilibrium distribution function. In this paper, we apply the D2Q21 model for two-dimensional flows and the D3Q39 model for three-dimensional flows proposed by Takada and Tsutahara [9]. The particle velocity vector is shown in Tables 1 and 2. The equilibrium distribution function is given by a following polynomial of the flow velocity up to the third order.

$$
\begin{aligned}
f_{i}^{(0)}= & F_{i} \rho\left(1-2 B c_{i \alpha} u_{\alpha}+2 B^{2} c_{i \alpha} c_{i \beta} u_{\alpha} u_{\beta}+B u^{2}\right. \\
& \left.-2 B^{2} c_{i \alpha} u_{\alpha} u^{2}-\frac{4}{3} B^{3} c_{i \alpha} c_{i \beta} c_{i \gamma} u_{\alpha} u_{\beta} u_{\gamma}\right)
\end{aligned}
$$

where $\rho, \boldsymbol{u}$ and $e$ denote the density, the flow velocity and the internal energy, respectively. They are defined by the particle velocity and the distribution function as follows.

$$
\begin{gathered}
\rho(\mathbf{x}, t)=\sum_{i} f_{i}(\mathbf{x}, t) \\
\rho(\mathbf{x}, t) u_{\alpha}(\mathbf{x}, t)=\sum_{i} f_{i}(\mathbf{x}, t) c_{i \alpha} \\
\rho(\mathbf{x}, t)\left(e(\mathbf{x}, t)+\frac{u(\mathbf{x}, t)^{2}}{2}\right)=\sum_{i} f_{i}(\mathbf{x}, t) \frac{c_{i}^{2}}{2}
\end{gathered}
$$

The coefficients $F_{i}$ and $B$ in equation (2) depend on the internal energy. They are determined to satisfy following constraints in order to recover the compressible Navier-Stokes equations. The coefficients $F_{i}$ and $B$ are presented in Tsutahara et al [13].

$$
\begin{gathered}
\sum_{i} f_{i}^{(0)}=\rho \\
\sum_{i} f_{i}^{(0)} c_{i \alpha}=\rho u_{\alpha} \\
\sum_{i} f_{i}^{(0)} c_{i \alpha} c_{i \beta}=\rho\left(e \delta_{\alpha \beta}+u_{\alpha} u_{\beta}\right)
\end{gathered}
$$




$$
\begin{gathered}
\sum_{i} f_{i}^{(0)} \frac{c_{i}^{2}}{2}=\rho\left(e+\frac{u^{2}}{2}\right) \\
\sum_{i} f_{i}^{(0)} c_{i \alpha} \frac{c_{i}^{2}}{2}=\rho u\left(2 e+\frac{u^{2}}{2}\right)
\end{gathered}
$$

Table 1: $\quad$ Velocity set in D2Q21 model.

\begin{tabular}{|c|c|c|}
\hline$i$ & Velocity vector & $|\mathbf{c}|$ \\
\hline 1 & $(0,0)$ & 0 \\
\hline $2-5$ & $(1,0),(0,1),(-1,0),(0,-1)$ & 1 \\
\hline $6-9$ & $(2,0),(0,2),(-2,0),(0,-2)$ & 2 \\
\hline $10-13$ & $(3,0),(0,3),(-3,0),(0,-3)$ & 3 \\
\hline $14-17$ & $(1,1),(-1,1),(-1,-1),(1,-1)$ & $\sqrt{2}$ \\
\hline $18-21$ & $(2,2),(-2,2),(-2,-2),(2,-2)$ & $2 \sqrt{2}$ \\
\hline
\end{tabular}

Table 2: $\quad$ Velocity set in 3D39V model.

\begin{tabular}{|c|c|c|}
\hline$i$ & Velocity vector & $\mid \mathbf{c}$ \\
\hline 1 & $(0,0,0)$ & 0 \\
\hline $2-7$ & $\begin{array}{c}(1,0,0),(-1,0,0),(0,1,0), \\
(0,-1,0),(0,0,1),(0,0,-1)\end{array}$ & 1 \\
\hline $8-13$ & $\begin{array}{c}(2,0,0),(-2,0,0),(0,2,0), \\
(0,-2,0),(0,0,2),(0,0,-2)\end{array}$ & 2 \\
\hline $14-19$ & $\begin{array}{c}(3,0,0),(-3,0,0),(0,3,0), \\
(0,-3,0),(0,0,3),(0,0,-3)\end{array}$ & 3 \\
\hline $20-31$ & $\begin{array}{c}(2,2,0),(-2,2,0),(-2,-2,0),(2,-2,0), \\
(0,2,2),(0,-2,2),(0,-2,-2),(0,2,-2), \\
(2,0,2),(-2,0,2),(-2,0,-2),(2,0,-2)\end{array}$ & $2 \sqrt{2}$ \\
\hline $32-39$ & $\begin{array}{c}(1,1,1),(-1,1,1),(-1,-1,1),(1,-1,1), \\
(1,1,-1),(-1,1,-1),(-1,-1,-1),(1,-1,-1)\end{array}$ & $\sqrt{3}$ \\
\hline
\end{tabular}

\section{Arbitrary Lagrangian Eulerian (ALE) formulation}

The ALE method is generally used to simulate flows around moving bodies or to calculate the problem that contains interface of multi-phase flows. In the ALE method, a flow-field is described by the Eulerian formulation, and a grid moves together with moving boundaries. In this paper, a grid deformation is not considered and the grid only translates or rotates as a solid body. Thus, we can easily present a formulation of the ALE method, where the convection velocity of the equation is replaced with the relative velocity between the convection velocity and the grid velocity. On the surface of moving boundary, a flow velocity is given by that of the moving body as the boundary condition. 
If the discrete BGK equation is regarded as a convection equation, the particle velocity corresponds to a convection velocity. Thus, the discrete BGK equation is reformulated as follows [18].

$$
\begin{aligned}
& \frac{\partial f_{i}(\mathbf{x}, t)}{\partial t}+\left\{c_{i \alpha}-V_{\alpha}(\mathbf{x}, t)\right\} \frac{\partial f_{i}(\mathbf{x}, t)}{\partial x_{\alpha}}-\frac{A}{\phi} c_{i \alpha} \frac{\partial\left\{f_{i}(\mathbf{x}, t)-f_{i}^{(0)}(\mathbf{x}, t)\right\}}{\partial x_{\alpha}} \\
& =-\frac{1}{\phi}\left\{f_{i}(\mathbf{x}, t)-f_{i}^{(0)}(\mathbf{x}, t)\right\}
\end{aligned}
$$

where $\boldsymbol{V}$ is the grid velocity vector. The third term of the left hand side means negative viscosity. We can set large time increment in high Reynolds number flows due to this additional term [19]. As a result, computational time becomes shorter. This term is not replaced although it includes a particle velocity, because this term represents the negative viscosity and is not a convection term.

By applying the Chapman-Enskog expansion, we obtain the Navier-Stokes equations

$$
\begin{gathered}
\frac{\partial \rho}{\partial t}+\frac{\partial}{\partial x_{\alpha}}\left\{\rho\left(u_{\alpha}-V_{\alpha}\right)\right\}=0 \\
\frac{\partial}{\partial t}\left(\rho u_{\alpha}\right)+\frac{\partial}{\partial x_{\beta}}\left\{\rho u_{\alpha}\left(u_{\beta}-V_{\beta}\right)+p \delta_{\alpha \beta}\right\} \\
-\frac{\partial}{\partial x_{\beta}}\left\{\mu\left(\frac{\partial u_{\beta}}{\partial x_{\alpha}}+\frac{\partial u_{\alpha}}{\partial x_{\beta}}\right)+\lambda \frac{\partial u_{\gamma}}{\partial x_{\gamma}} \delta_{\alpha \beta}\right\}=0 \\
\frac{\partial}{\partial t}\left\{\rho\left(e+\frac{u^{2}}{2}\right)\right\}+\frac{\partial}{\partial x_{\alpha}}\left\{\rho\left(u_{\alpha}-V_{\alpha}\right)\left(e+\frac{u^{2}}{2}\right)+p u_{\alpha}\right\} \\
-\frac{\partial}{\partial x_{\alpha}}\left\{\kappa^{\prime} \frac{\partial e}{\partial x_{\alpha}}+\mu u_{\beta}\left(\frac{\partial u_{\beta}}{\partial x_{\alpha}}+\frac{\partial u_{\alpha}}{\partial x_{\beta}}\right)+\lambda u_{\alpha} \frac{\partial u_{\beta}}{\partial x_{\beta}}\right\}=0
\end{gathered}
$$

where the pressure, the viscosity, the second viscosity, and the thermal diffusivity are expressed as follows

$$
p=\rho e, \mu=\rho e(\phi-A), \lambda=-\rho e(\phi-A)=-\mu, \kappa^{\prime}=2 \rho e(\phi-A)
$$

The sound speed is given as

$$
c_{s}=\sqrt{2 e}
$$

\subsection{Sound emitted from a rapidly rotating elliptic cylinder and the grid system}

The grid system consists of a boundary fitted co-ordinate system near the elliptic cylinder which rotates with the cylinder and outside of which is a cylindrical coordinates at rest as shown in Fig.1. Between the two grid systems, we set a buffer region and variables are connected through the region. 

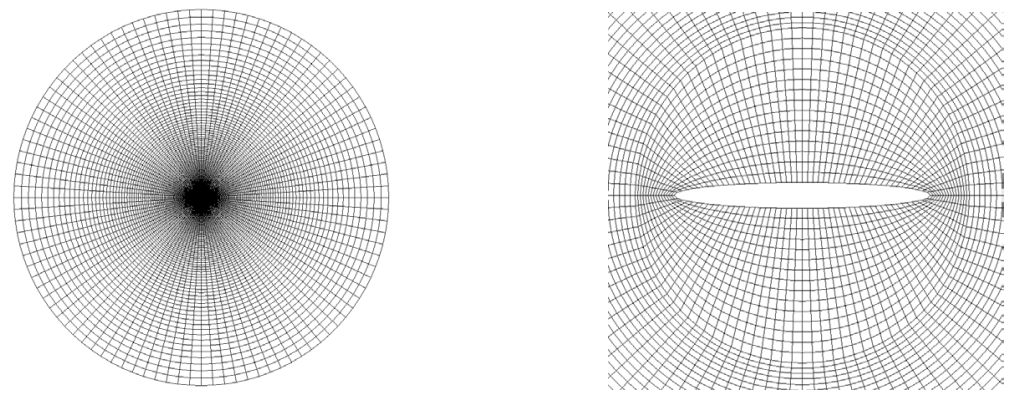

Figure 1: Grid for computation.

\subsection{Sound pressure field and sound source}

The Reynolds number, the Mach number and the aspect ratio are nondimensional parameters of this problem and they are defined as follows.

$$
R e=\frac{\rho U L}{\mu}, M a=\frac{U}{c_{s}}, A r=\frac{L}{l}
$$

where $U$ is the peripheral velocity of the edge of the elliptic cylinder, $L$ is the chord length, $l$ is the thickness.

We show the result for $\mathrm{Ar}=10.0, \mathrm{Re}=10000$ and $\mathrm{Ma}=0.2$. The number of the grid is $(r \times \theta)=(421 \times 301)$. The streamlines are shown in Fig. 2(a) and two vortices appear and rotate slowly in the same direction as the cylinder rotation. The pressure field in the vicinity of the cylinder at the same time is shown in Fig.2(b). When the edge of the cylinder passes through the vortices and goes into the potential region, the pressure on the front side of the edge rise sharply and on the other side the pressure drops and this pressure change is the sound source. The sound pressure fields when the sound is emitted are shown in Fig.3.

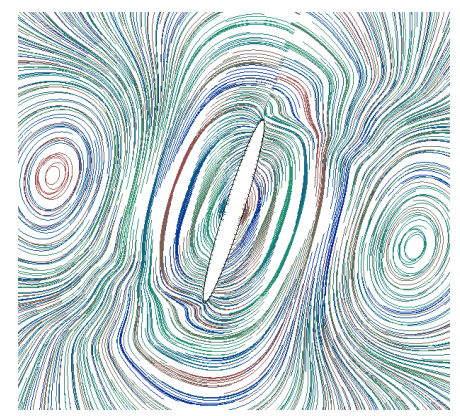

(a)

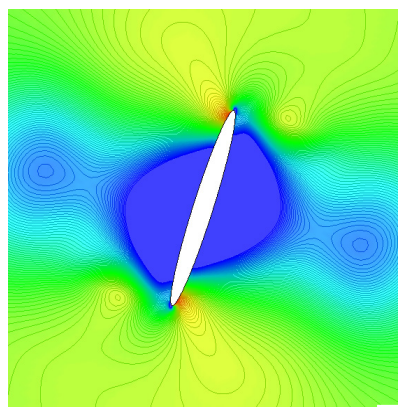

(b)

Figure 2: $\quad$ Stream-lines and pressure distributions at non-dimensional time $\mathrm{t}^{*}$ $=6.24$. 


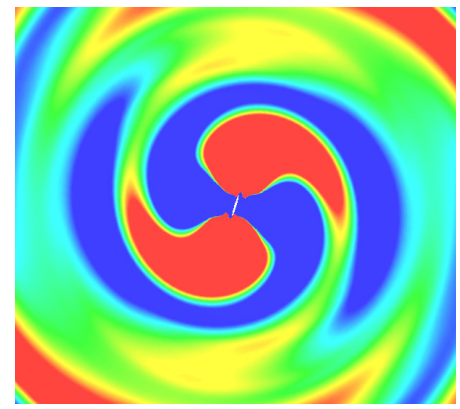

(a) $t^{*}=6.24$

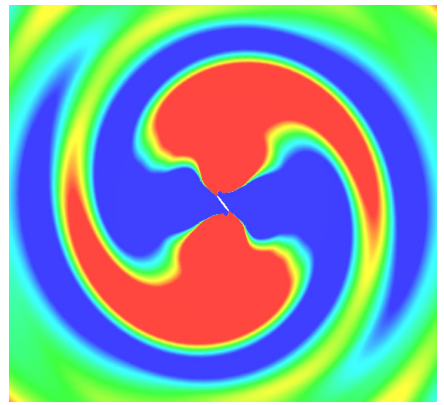

(b) $\mathrm{t}^{*}=6.40$

Figure 3: $\quad$ Sound pressure field near the elliptic cylinder.

\section{Two-phase flow model}

As a two-phase, liquid and gas, model, Swift et al have presented models employing free energy and they are widely used. But it is difficult to extend these models to a real liquid and gas model.

We use a two-particle model in this paper. Two particle distribution functions are defined for, say, blue and red particles, and are written as $f_{b}$ and $f_{r}$, respectively. Macroscopic variables are defined in (3) (4) and (5) for each fluid, but the fluid velocity is common to both fluids and the internal energy for liquid is neglected.

\subsection{A model for liquid}

The liquid has density much larger than that of the gas and also has larger elasticity, but it is difficult to realize these properties by the LBM because the LBM model is for an ideal gas.

For large elasticity model, we introduce a new definition of pressure, which has a relation with the bulk elasticity as

$$
P^{\prime}=P+\beta\left(\rho_{b}-\rho_{b, r e f}\right)
$$

where $\rho_{b, r e f}$ is the reference density defined appropriately. Then the sound speed in liquid is given as

$$
c_{s}=\sqrt{\frac{\Delta P^{\prime}}{\Delta \rho}} \sim \sqrt{\beta}
$$

and this sound speed is shown to be realized properly.

\subsection{Two-phase flow model with large density difference}

For two-phase, liquid and gas, flow models with large density, He et al. [20] introduce a model with the density difference up to 20 or so. They introduce two distribution functions, and one is only used as an index function that is detected the position of the interface. 
Inamuro et al. [21] also present a model with the density difference up to 1000 , but in their model the velocity field and the pressure field must be recalculated because the densities are uniformly defined in liquid and gas phases.

We present novel model including the density difference. The idea is very simple, that is the effect of the density difference appears the difference of the acceleration of the fluid acted some force. Therefore, we can consider the density difference by changing the acceleration. If the density of liquid, corresponding to the blue particles, is $\mathrm{m}$ times larger than that of gas, corresponding the red particles, the decelerations of the gas and liquid are

$$
\begin{aligned}
& \mathbf{a}_{\mathrm{mod}, r}=-\left(-\frac{1}{\rho} \nabla P+\frac{\mu}{\rho} \nabla^{2} \mathbf{u}\right)+\frac{1}{m_{r}}\left(-\frac{1}{\rho} \nabla P+\frac{\mu}{\rho} \nabla^{2} \mathbf{u}\right)+\tau g \\
& \mathbf{a}_{\mathrm{mod}, b}=-\left(-\frac{1}{\rho} \nabla P+\frac{\mu}{\rho} \nabla^{2} \mathbf{u}\right)+\frac{1}{m_{b}}\left(-\frac{1}{\rho} \nabla P^{\prime}+\frac{\mu}{\rho} \nabla^{2} \mathbf{u}\right)+\tau g
\end{aligned}
$$

in which $m_{r}$ and $m_{b}$ are the masses of the gas and the liquid particles, respectively, and the last terms in (20) and (21) are effect of the gravitational force.

\subsection{Phase separation and surface tension}

\subsubsection{Shang-Chen model}

The surface tension is defined by the gradient of blue particles, and the velocity for determining the local equilibrium distribution function is changed by the fluid velocity due to the impulse of the surface tension force [16,17] as

$$
\begin{aligned}
& \mathbf{u}_{b}=\mathbf{u}+\tau \times \mathbf{a}_{\text {mod, } b}+\tau \times \kappa \rho^{r} \nabla \rho^{b} \\
& \mathbf{u}_{r}=\mathbf{u}+\tau \times \mathbf{a}_{\mathrm{mod}, r}-\tau \times \kappa \rho^{b} \nabla \rho^{b}
\end{aligned}
$$

where $\kappa$ represent the strength of the surface tension. By substituting the above mentioned velocities to the local equilibrium distribution function, a two-phase fluid model with large density difference is obtained.

\subsubsection{Latva-Kokko and Rothman model}

In order to separate the liquid and the gas phases, the surface tension force is commonly used as the above mentioned Shang-Chen model, but this technique gives large spurious velocities. Then we use a method proposed by Latva-Kokko and Rothman [22], in which the distribution of particles is separated on the liquid side and the gas side on the interface.

$$
\begin{aligned}
& f_{i L}^{\prime}=\frac{\rho_{L}}{\rho_{L}+\rho_{G}}\left(f_{i L}+f_{i G}\right)-\kappa_{1} \frac{\rho_{L} \rho_{G}}{\left(\rho_{L}+\rho_{G}\right)^{2}}\left(f_{i L}^{e q(0)}+f_{i G}^{e q(0)}\right) \cos \varphi \\
& f_{i G}^{\prime}=\frac{\rho_{G}}{\rho_{L}+\rho_{G}}\left(f_{i L}+f_{i G}\right)+\kappa_{1} \frac{\rho_{L} \rho_{G}}{\left(\rho_{L}+\rho_{G}\right)^{2}}\left(f_{i L}^{e q(0)}+f_{i G}^{e q(0)}\right) \cos \varphi
\end{aligned}
$$

where $\kappa_{1}$ is a factor of particle separation on the interface, $f_{i L}^{e q(0)}$ is a local equilibrium distribution function for velocity 0 , and $\varphi$ represents the angle of the interface. 
The surface tension is defined as a normal force to the interface, and the force is proportional to the radius of curvature as

$$
F_{s \alpha}=\sigma \kappa n_{\alpha}
$$

where $\sigma$ is the radius of curvature, $\kappa$ is the surface tension, and $n_{\alpha}$ is a normal unit vector on the interface. By substituting the above mentioned velocities to the local equilibrium distribution function, a two-phase fluid model with large density difference is obtained.

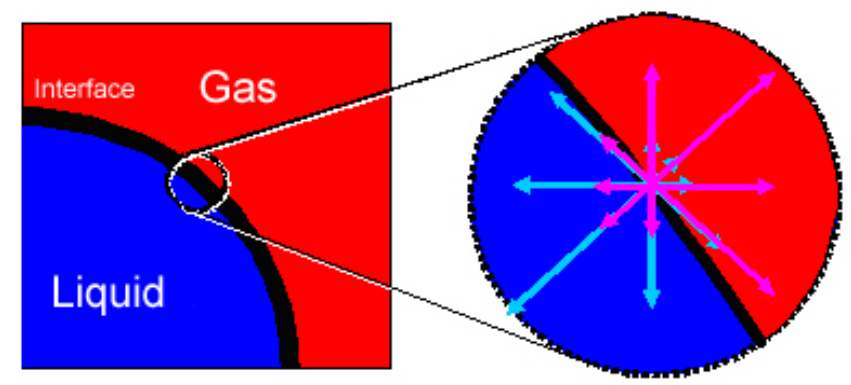

Figure 4: $\quad$ Phase separation model of Latova-Kokko and Rothman.

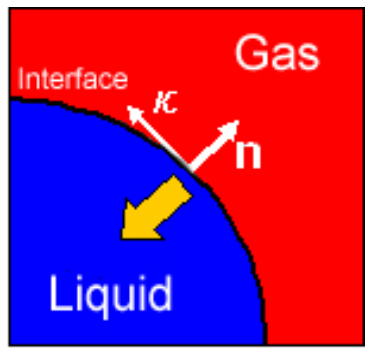

Figure 5: $\quad$ Surface tension model.

\subsection{Water drop colliding the water surface and generated sound}

Figure 6 shows the sound generated by the collision of a 3D water drop with a water surface. Calculation parameters are as follows: the factor of particle separation $\kappa_{1}=2.0$, the surface tension $\kappa=0.004$, the bulk elasticity $\beta=16000$, and the acceleration of gravity $g=0.001$. In (a) the shape of the drop at the collision and the shadow graph of the sound generated on the interface are shown. In (b) the shape of the splash and the its shadow graph are shown. The sound propagating in the air and also the under water sound are clearly seen.

\section{Conclusions}

Direct simulations of fluid dynamic sound are performed by the finite difference lattice Boltzmann method. The Arbitrary Lagrangian Eulerian formulation is 
applied to the simulation of sound emitted from a rapidly rotating elliptic cylinder and the sound source is detected. A two-phase flow model with large density difference and including the elasticity of liquid is proposed and a simulation of sound generated when a water drop collides with a water surface is also performed.

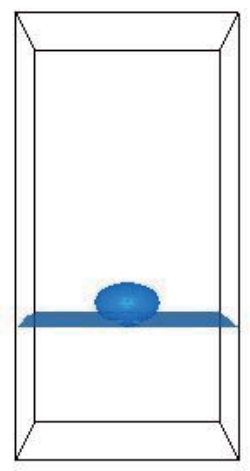

(a)
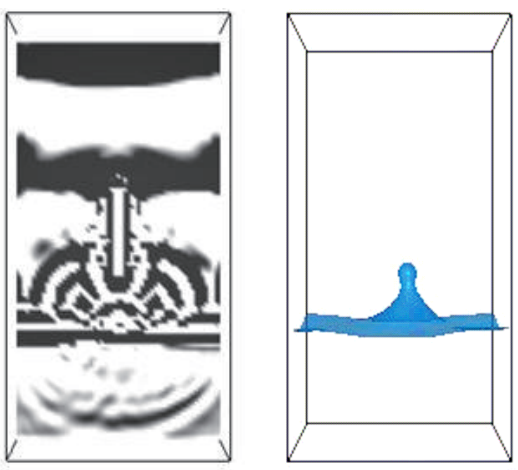

(b)

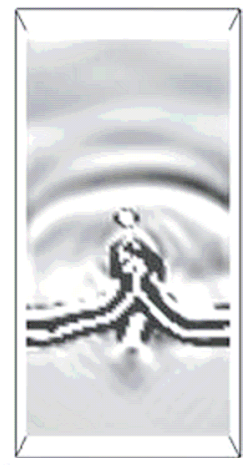

Figure 6: Sound generated at the collision of a water drop with a water surface.

\section{References}

[1] Qian, Y.H., Succi, S. and Orszag, S.A., Recent Advances in Lattice Boltzmann Computing, Ann. Rev. of Comp. Phy. III, D. Stauffer ed. World Scientific, pp.195-242, 1995.

[2] Rothman, D.H. and Zalenski, S., Lattice-Gas Cellular Automata, Cambridge U.P., 1997.

[3] Chopard, B. and Droz, M., Cellular Automata Modeling of Physical Systems, Cambridge University Press, 1998.

[4] Chen, S. and Doolen, G.D., Lattice Boltzmann method for fluid flows, Ann. Rev. Fluid Mech., Ann. Rev. Inc. pp.329-364, 1998.

[5] Wolf-Gladrow, D.A., Lattice-Gas Cellular Automata and Lattice Boltzmann Models, Lecture Notes in Mathematics, Springer, 2000.

[6] Succi, S., The lattice Boltzmann Equation for Fluid Dynamics and Beyond, Oxford, 2001.

[7] Alexander, F.J. et al., Lattice Boltzmann thermodynamics, Phys. Rev. E, 47 R2249-R2252, 1993.

[8] Chen, Y., et al., Thermal lattice Bhatanagar Gross Knook model without nonlinear deviations in macrodynamic equations, Phys. Rev. E, 50, pp.2776-2283, 1994.

[9] Takada, N. and Tsutahara M., Proposal of Lattice BGK model with internal degrees of freedom in lattice Boltzmann method, Transaction of JSME B, 65-629, pp.92-99, 1999 (in Japanese). 
[10] McNamara, G.R., et al., Stabilization of thermal lattice Boltzmann models, J. Stat. Phys., 81(1/2), pp. 395-408, 1995.

[11] Kataoka, T. and Tsutahara, T., Lattice Boltzmann model for the compressible Navier-Stokes equations with flexible specific-heat ratio, Phys. Rev. E, 67, pp.036306-1-4, 2004.

[12] Watari, M. and Tsutahara, T., Two-dimensional thermal model of the finite-difference lattice Boltzmann method with high spatial isotropy, Phys. Rev. E, 69, pp.035701-1-7, 2004.

[13] Tsutahara M, Takada N, Kataoka T, Lattice gas and lattice Boltzmann methods, Corona-sha, (in Japanese) 1999.

[14] Swift, M.R., Orlandini, E., Osborn, W.R., and Yeomans, J.M., Lattice Boltzmann simulations of liquid-gas and binary-fluid systems. Phys. Rev. E 54, pp.5041-5052, 1996.

[15] Swift, M.R., Osborn, W.R., and Yeomans, J.M., Lattice Boltzmann simulation of non-ideal fluids. Phys. Rev. Lett. 75, pp.830-833, 1995.

[16] Shan, X. and Chen, H., Lattice Boltzmann model for simulating flows with multiple phases and components. Phys. Rev. E 47, pp.1815-1819, 1993.

[17] Shan, X. and Chen, H. Simulation of non-ideal gases and liquid-gas phase-transitions by the lattice Boltzmann-equation. Phys. Rev. E 49, pp.2941-2948, 1994.

[18] Tamura, A., Tsutahara, M., Matsuoka. H. Direct simulation of bladevortex interaction by the finite difference lattice Boltzmann method, WESPAC 2006 CD-ROM, 2006.

[19] Tsutahara, M., Kurita, M., and Kataoka, T., Direct simulation of Aeolian tone by the finite difference Lattice Boltzmann method, Computational Fluid Dynamics 2002, pp.508-513, 2003.

[20] He X., Chen S., and Zhang R., A lattice Boltzmann Scheme for Incompressible Multiphase Flow and Its Application in Simulation of Rayleigh-Taylor Instability, J. Computational Physics 152, pp.642-663, 1999.

[21] Inamuro T., Ogata T., Tajima S. and Konishi N., A lattice Boltzmann method for incompressible two-phase flows with large density differences, J. Computational Physics 198, pp.628-644, 2004.

[22] Latva-Kokko, M. and Rothman D.H., Diffusion Properties of Gradientbased Lattice Boltzmann Models of Immiscible Fluids, Physical Review E, 71 pp. 056702 1-8, 2005. 